\title{
Modeling and qualitative analysis of malaria epidemiology
}

\author{
Abdulrahman Ndanusa ${ }^{1}$, Aminatu Abimbola Busari ${ }^{2}$ \\ ${ }^{1,2}$ (Department of Mathematics \& Statistics, Federal University of Technology, Minna, Nigeria)
}

\begin{abstract}
We develop and analyze a mathematical model on malaria dynamics using ordinary differential equations, in order to investigate the effect of certain parameters on the transmission and spread of the disease. We introduce dimensionless variables for time, fraction of infected human and fraction of infected mosquito and solve the resulting system of ordinary differential equations numerically. Equilibrium points are established and stability criteria analyzed. The results reveal that the parameters play a crucial role in the interaction between human and infected mosquito.
\end{abstract}

Keywords - Endemic, Epidemic, Epidemiology, Equilibrium, Malaria

\section{Introduction}

Malaria is a parasitic vector-borne disease caused by a protozoan parasite of the genus Plasmodium. The disease is transmitted to humans by a mosquito of the genus Anopheles. Each time an Anopheles mosquito bites an infected person, it becomes infected with the malaria parasite. Thereafter, the malaria parasites replicate inside the mosquito's body. The mosquito then retransmits the parasites to anyone that it bites through its saliva. The parasites undergo series of transformations inside the human body until malaria symptoms become apparent in about seven to twenty one days after infection. Malaria is a serious disease which if left untreated could result in death. According to World Health Organization (WHO) estimates malaria is responsible for almost one million deaths a year in Sub-Saharan Africa. However, malaria is a disease that can be prevented, if standard and adequate measures are put in place, or contained, leading to an outbreak of the disease in small proportion (epidemic), or even widespread outbreak of the disease (endemic).

The first attempt at mathematical modeling of malaria is attributed to Ross [1]. A major extension of Ross' model was carried out by Macdonald [2]. In its simplest form, the Ross-Macdonald model has the representation:

$$
\begin{aligned}
& \frac{d x}{d t}=a b \frac{M}{N} y(1-x)-r x \\
& \frac{d y}{d t}=a x(1-y)-\mu y
\end{aligned}
$$

Ever since, there has been various modifications, improvements and advancements in mathematical modeling of malaria. In their model, Aron and May [3] considered some malaria characteristics such as an incubation period in the mosquito, a periodically fluctuating density of mosquitoes, superinfection and a period of immunity in humans. Anderson and May [4] considered the effect of age structure on the basic RossMacdonald model. Roberts and Heesterbeek [5] gave an overview of the use of mathematical models to explain the epidemiology of infectious diseases, and to assess the potential benefits of proposed control strategies with special reference to malaria. Chitnis [6] modeled malaria using ordinary differential equations. He analyzed the existence and stability of disease-free and endemic equilibria.

This is an attempt to investigate the effects of certain parameters involved in the dynamics of malaria epidemiology through mathematical modeling, with the aim of describing the distribution of the disease, identifying the risk factors, for the disease, and providing a source of information for planners and administrators involved in various prevention, detection and control programmes.

\section{Materials and Methods}

Following Roberts and Heesterbeek [5] we analyze a simple malaria model as follows:

$$
\left.\begin{array}{ll}
\frac{d X}{d \tau}=a b \frac{M}{N} Y(1-X)-\gamma X & X(0)=X_{0} \\
\frac{d Y}{d \tau}=a c X(1-Y)-\mu Y & Y(0)=Y_{0}
\end{array}\right\}
$$

where

$X=$ Fraction of infected human.

$Y=$ Fraction of infected mosquitos.

$\frac{M}{N}=$ Number of female mosquitoes per human host in an infection free state. 
$a=$ Per biting rate of mosquitoes on humans.

$b=$ Probability that a bite by an infectious mosquito transmits the agent.

$c=$ Probability that a bite of an infected human by a susceptible mosquito results in transmission of the agent to the mosquito.

$\gamma=$ Rate at which humans recover from infection.

$\mu=$ Per capita death rate of mosquitos.

We introduce dimensionless variable for fraction of infected human, fraction of infected mosquitos and time. That is, we let

$$
\begin{aligned}
x & =\frac{a c}{\mu} X \\
y & =\frac{a b M}{\gamma N} Y \\
t & =a c t
\end{aligned}
$$

Then

$X=\frac{\mu}{a c} x$

$d X=\frac{\mu}{a c} x d x$

$Y=\frac{\gamma N}{a b M} y$

$d Y=\frac{\gamma N}{a b M} d y$

$\tau=\frac{1}{a c} t$

$d \tau=\frac{1}{a c} d t$

Substituting (7) - (12) into the first equation of (3) gives:

$\mu \frac{d x}{d t}=\frac{a b M}{N} \frac{\gamma N}{a b M} y\left(1-\frac{\mu}{a c} x\right)-\frac{\gamma \mu}{a c} x$

$\frac{d x}{d t}=\frac{\gamma}{\mu} y\left(1-\frac{\mu}{a c} x\right)-\frac{\gamma}{a c} x$

$\frac{d x}{d t}==\frac{\gamma}{\mu} y-\frac{\gamma}{a c} x y-\frac{\gamma}{a c} x$

Similarly, on substituting (7) - (12) into the second equation of (3) gives:

$\frac{\gamma N a c}{a b M} \frac{d y}{d t}=\frac{a c \mu}{a c} x\left(1-\frac{\gamma N}{a b M} y\right)-\mu \frac{\gamma N}{a b M} y$

$\frac{d y}{d t}=\frac{a b M}{\gamma N a c} \frac{a c \mu}{a c} x\left(1-\frac{\gamma N}{a b M} y\right)-\frac{a b M}{\gamma N a c} \mu \frac{\gamma N}{a b M} y$

$\frac{d y}{d t}=\frac{b M \mu}{\gamma N c} x\left(1-\frac{\gamma N}{a b M} y\right)-\frac{\mu}{a c} y$

$\frac{d y}{d t}=\frac{b M \mu}{\gamma N c} x-\frac{\mu}{a c} x y-\frac{\mu}{a c} y$

Therefore, the non-dimensionalized equations are

$\left.\begin{array}{l}\frac{d x}{d t}=\alpha y-\beta x(1+y) \\ \frac{d y}{d t}=\sigma x-v y(1+x)\end{array}\right\}$

Where

$\alpha=\frac{\gamma}{\mu}$ is the human recovery rate.

$\beta=\frac{Y}{a c}$ is the per capita biting rate of mosquitoes on human.

$\sigma=\frac{b M \mu}{\gamma N c}$ is the number of female mosquitoes per human host in an infection free steady state.

$v=\frac{\underline{\mu}}{a c}$ is the per capita death rate of mosquitoes. 


\subsection{Equilibria}

Disease free equilibrium:- This is the equilibrium that is attained by an entirely healthy population in the absence of mosquitoes:

$x=x^{*}, \quad y=0$

$P_{1}=\left(x^{*}, 0\right)$

Endemic equilibrium:- At this state, the human contains the susceptible and infected. The equilibrium generally takes the form:

$x=x^{* *}, \quad y=y^{* *}$
$P_{2}=\left(x^{* *}, y^{* *}\right)$

\subsection{Stability Analysis}

The jacobian matrix of (20) is represented by:

$l_{F}(x, y)=\left(\begin{array}{cc}-\beta(1+y) & \alpha-\beta x \\ \sigma-v y & -v(1+x)\end{array}\right)$

The linearization of equation $(20)$ at $P_{1}=\left(x^{*}, 0\right)$ is:

$l_{F}\left(x^{*}, 0\right)=\left(\begin{array}{cc}-\beta & \delta \\ \sigma & -\tau\end{array}\right)$

where

$\delta=\alpha-\beta x^{*}$

$\tau=v\left(1+x^{*}\right)$

The eigenvalue relation of (26) is:

$(\beta+\lambda)(\tau+\lambda)-\sigma \delta=0$

$\lambda^{2}+(\beta+\tau) \lambda+(\beta \tau-\sigma \delta)=0$

Thus,

$\lambda=\frac{-(\beta+\tau) \pm \sqrt{(\beta+\tau)^{2}-4(\beta \tau-\sigma \delta)}}{2}$

$\lambda_{1}=\frac{-(\beta+\tau)+\sqrt{(\beta+\tau)^{2}-4(\beta \tau-\sigma \delta)}}{2}$

$\lambda_{2}=\frac{-(\beta+\tau)-\sqrt{(\beta+\tau)^{2}-4(\beta \tau-\sigma \delta)}}{2}$

Table 2.1 Stability properties of linear systems

\begin{tabular}{lll}
\hline Eigenvalue & Type of critical point & Stability \\
$\lambda_{1}>\lambda_{2}>0$ & Improper node & Unstable \\
$\lambda_{1}<\lambda_{2}<0$ & Improper node & Asymptotically stable \\
$\lambda_{2}<0<\lambda_{1}$ & Saddle point & Unstable \\
$\lambda_{1}=\lambda_{2}>0$ & Proper or improper node & Unstable \\
$\lambda_{1}=\lambda_{2}<0$ & Proper or improper node & Asymptotically stable \\
$\lambda_{1}, \lambda_{2}=\lambda \pm \boldsymbol{i} \boldsymbol{\mu}$ & Spiral point & \\
$\lambda>0$ & & Unstable \\
$\lambda<0$ & & Asymptotically stable \\
$\lambda_{1}=\boldsymbol{i} \boldsymbol{\mu}, \lambda_{2}=-\boldsymbol{i} \boldsymbol{\mu}$ & Centre & Stable \\
\hline
\end{tabular}

Table 2.1 provides information on the type of stability that could result based on the nature of the eigenvalues of a linear system. By definition, all parameters are non-negative. Thus,

1. If $\beta \tau>\sigma \delta$ and $(\beta+\tau)^{2}-4(\beta \tau-\sigma \delta)>0$ the eigenvalues are real, unequal and negative. Hence, the critical point $\left(x^{*}, 0\right)$ is an asymptotically stable improper node of the system.

2. If $(\beta+\tau)^{2}-4(\beta \tau-\sigma \delta)=0$ the eigenvalues are negative. Hence, the critical point $\left(x^{*}, 0\right)$ is globally asymptotically stable.

3. If $\beta \tau>\sigma \delta$ and $(\beta+\tau)^{2}-4(\beta \tau-\sigma \delta)<0$ we have one negative root and two complex roots whose real parts are equal and negative. Hence, the critical point $\left(x^{*}, 0\right)$ is globally asymptotically stable.

The linearization of equation (20) at $P_{2}=\left(x^{* *}, y^{* *}\right)$ is: 
$l_{F}\left(x^{* *}, y^{* *}\right)=\left(\begin{array}{cc}-\beta_{1} & \delta_{1} \\ \sigma_{1} & -\tau_{1}\end{array}\right)$

where

$$
\begin{aligned}
& \beta_{1}=-\beta\left(1+y^{* *}\right) \\
& \delta_{1}=\alpha-\beta x^{* *} \\
& \sigma_{1}=\sigma-v y^{* *}
\end{aligned}
$$

$$
\tau_{1}=-v\left(1+x^{* *}\right)
$$

The eigenvalue equation of (34) is therefore:

$$
\begin{aligned}
& \left(\beta_{1}+\lambda\right)\left(\tau_{1}+\lambda\right)-\sigma_{1} \delta_{1}=0 \\
& \lambda^{2}+\left(\beta_{1}+\tau_{1}\right) \lambda+\left(\beta_{1} \tau_{1}-\sigma_{1} \delta_{1}\right)=0
\end{aligned}
$$

Which leads to:

$$
\begin{aligned}
& \lambda=\frac{-\left(\beta_{1}+\tau_{1}\right) \pm \sqrt{\left(\beta_{1}+\tau_{1}\right)^{2}-4\left(\beta_{1} \tau_{1}-\sigma_{1} \delta_{1}\right)}}{2} \\
& \lambda_{1}=\frac{-\left(\beta_{1}+\tau_{1}\right)+\sqrt{\left(\beta_{1}+\tau_{1}\right)^{2}-4\left(\beta_{1} \tau_{1}-\sigma_{1} \delta_{1}\right)}}{2} \\
& \lambda_{2}=\frac{-\left(\beta_{1}+\tau_{1}\right)-\sqrt{\left(\beta_{1}+\tau_{1}\right)^{2}-4\left(\beta_{1} \tau_{1}-\sigma_{1} \delta_{1}\right)}}{2}
\end{aligned}
$$

Thus,

1. If $\beta_{1} \tau_{1}>\sigma_{1} \delta_{1}$ and $\left(\beta_{1}+\tau_{1}\right)^{2}-4\left(\beta_{1} \tau_{1}-\sigma_{1} \delta_{1}\right)$ the eigenvalues are real, unequal and negative. Hence, the critical point $\left(x^{*}, 0\right)$ is an asymptotically stable improper node of the system.

2. If $\left(\beta_{1}+\tau_{1}\right)^{2}-4\left(\beta_{1} \tau_{1}-\sigma_{1} \delta_{1}\right)$ the eigenvalues are negative. Hence, the critical point $\left(x^{*}, 0\right)$ is globally asymptotically stable.

3. If $\beta_{1} \tau_{1}>\sigma_{1} \delta_{1}$ and $\left(\beta_{1}+\tau_{1}\right)^{2}-4\left(\beta_{1} \tau_{1}-\sigma_{1} \delta_{1}\right)$ we have one negative root and two complex roots whose real parts are equal and negative. Hence, the critical point $\left(x^{*}, 0\right)$ is globally asymptotically stable

\section{Results and Discussion}

System (20) is solved numerically using Runge-Kutta integration scheme with a modified version of Newton-Raphson shooting method with $\alpha_{s} \beta, v$ and $\sigma$ as prescribed parameters. The computations were effected with the aid of MAPLE software package.

Table 3.1 The numbers of infected mosquitoes $y(t)$ at time $t$ for different values of $\beta$ when

$$
\alpha=2, \sigma=0.5 \text { and } v=1
$$

\begin{tabular}{cccccccc}
\hline $\boldsymbol{t}$ & $\boldsymbol{\beta}=\mathbf{3}$ & $\boldsymbol{\beta}=\mathbf{2}$ & $\boldsymbol{\beta}=\mathbf{1}$ & $\boldsymbol{t}$ & $\boldsymbol{\beta}=\mathbf{3}$ & $\boldsymbol{\beta}=\mathbf{2}$ & $\boldsymbol{\beta}=\mathbf{1}$ \\
\hline $\mathbf{0}$ & 10 & 10 & 10 & & & & \\
$\mathbf{0 . 1}$ & 6.307438 & 7.028782 & 7.505982 & $\mathbf{1 . 1}$ & 0.680533 & 1.324564 & 1.74744 \\
$\mathbf{0 . 2}$ & 4.461663 & 5.688294 & 6.374458 & $\mathbf{1 . 2}$ & 0.600122 & 1.155204 & 1.533901 \\
$\mathbf{0 . 3}$ & 3.31832 & 4.727512 & 5.466857 & $\mathbf{1 . 3}$ & 0.535898 & 1.012702 & 1.350544 \\
$\mathbf{0 . 4}$ & 2.544652 & 3.964324 & 4.701035 & $\mathbf{1 . 4}$ & 0.484005 & 0.89233 & 1.192761 \\
$\mathbf{0 . 5}$ & 1.997105 & 3.342174 & 4.051184 & $\mathbf{1 . 5}$ & 0.441587 & 0.790231 & 1.056664 \\
$\mathbf{0 . 6}$ & 1.598968 & 2.830669 & 3.498951 & $\mathbf{1 . 6}$ & 0.406512 & 0.703259 & 0.938978 \\
$\mathbf{0 . 7}$ & 1.303937 & 2.408395 & 3.02918 & $\mathbf{1 . 7}$ & 0.377173 & 0.628842 & 0.836943 \\
$\mathbf{0 . 8}$ & 1.082003 & 2.058739 & 2.62911 & $\mathbf{1 . 8}$ & 0.352354 & 0.564877 & 0.748233 \\
$\mathbf{0 . 9}$ & 0.912858 & 1.768393 & 2.287961 & $\mathbf{1 . 9}$ & 0.331126 & 0.509642 & 0.670887 \\
$\mathbf{1}$ & 0.782375 & 1.526586 & 1.996632 & $\mathbf{2}$ & 0.312777 & 0.461721 & 0.603251 \\
\hline
\end{tabular}

It is observed that the population of infected mosquitoes, $y(t)$, increases as per capita biting rate of mosquitoes on human, $\beta$, decreases. 
Table 3.2 The numbers of infected human $x(t)$ at time $t$ for different values of $\beta$ when $\alpha=2, \sigma=0.5$ and $v=1$

\begin{tabular}{cccccccc}
\hline $\boldsymbol{t}$ & $\boldsymbol{\beta}=\mathbf{3}$ & $\boldsymbol{\beta}=\mathbf{2}$ & $\boldsymbol{\beta}=\mathbf{1}$ & $\boldsymbol{t}$ & $\boldsymbol{\beta}=\mathbf{3}$ & $\boldsymbol{\beta}=\mathbf{2}$ & $\boldsymbol{\beta}=\mathbf{1}$ \\
\hline $\mathbf{0}$ & 5 & 5 & 5 & & & & \\
$\mathbf{0 . 1}$ & 3.096693 & 1.537987 & 0.851371 & $\mathbf{1 . 1}$ & 1.22183 & 0.632655 & 0.445965 \\
$\mathbf{0 . 2}$ & 2.433385 & 1.01735 & 0.60548 & $\mathbf{1 . 2}$ & 1.154859 & 0.603486 & 0.427726 \\
$\mathbf{0 . 3}$ & 2.106858 & 0.888508 & 0.572797 & $\mathbf{1 . 3}$ & 1.092208 & 0.574555 & 0.409282 \\
$\mathbf{0 . 4}$ & 1.905678 & 0.837103 & 0.557788 & $\mathbf{1 . 4}$ & 1.033643 & 0.546068 & 0.390767 \\
$\mathbf{0 . 5}$ & 1.760408 & 0.804022 & 0.543965 & $\mathbf{1 . 5}$ & 0.978959 & 0.518206 & 0.372313 \\
$\mathbf{0 . 6}$ & 1.643581 & 0.775415 & 0.529506 & $\mathbf{1 . 6}$ & 0.927963 & 0.491123 & 0.354039 \\
$\mathbf{0 . 7}$ & 1.543002 & 0.747561 & 0.514207 & $\mathbf{1 . 7}$ & 0.880461 & 0.464943 & 0.336055 \\
$\mathbf{0 . 8}$ & 1.45284 & 0.719433 & 0.498103 & $\mathbf{1 . 8}$ & 0.836257 & 0.43976 & 0.318458 \\
$\mathbf{0 . 9}$ & 1.370161 & 0.690833 & 0.481284 & $\mathbf{1 . 9}$ & 0.795153 & 0.415644 & 0.30133 \\
$\mathbf{1}$ & 1.293436 & 0.661844 & 0.463863 & $\mathbf{2}$ & 0.756952 & 0.392638 & 0.28474 \\
\hline
\end{tabular}
increases.

The population of infected human, $x(t)$, increases as per capita biting rate of mosquitoes on human, $\beta$,

Table 3.2 The numbers of infected mosquitoes $y(t)$ at time $t$ for different values of $\alpha$ when $\beta=1_{x} \sigma=0.5$ and $v=1$

\begin{tabular}{cccccccc}
\hline $\boldsymbol{t}$ & $\boldsymbol{\alpha}=\mathbf{2}$ & $\boldsymbol{\alpha}=\mathbf{4}$ & $\boldsymbol{\alpha}=\mathbf{6}$ & $\boldsymbol{t}$ & $\boldsymbol{\alpha}=\mathbf{2}$ & $\boldsymbol{\alpha}=\mathbf{4}$ & $\boldsymbol{\alpha}=\mathbf{6}$ \\
\hline $\mathbf{0}$ & 10 & 10 & 10 & & & & \\
$\mathbf{0 . 1}$ & 6.307438 & 5.946536 & 5.612748 & $\mathbf{1 . 1}$ & 0.782375 & 0.508089 & 0.440167 \\
$\mathbf{0 . 2}$ & 4.461663 & 3.784062 & 3.238716 & $\mathbf{1 . 2}$ & 0.600122 & 0.429714 & 0.403296 \\
$\mathbf{0 . 3}$ & 3.31832 & 2.525192 & 1.976655 & $\mathbf{1 . 3}$ & 0.535898 & 0.404368 & 0.391487 \\
$\mathbf{0 . 4}$ & 2.544652 & 1.76302 & 1.298392 & $\mathbf{1 . 4}$ & 0.484005 & 0.38454 & 0.382079 \\
$\mathbf{0 . 5}$ & 1.997105 & 1.28908 & 0.925809 & $\mathbf{1 . 5}$ & 0.441587 & 0.368601 & 0.374285 \\
$\mathbf{0 . 6}$ & 1.598968 & 0.987431 & 0.7153 & $\mathbf{1 . 6}$ & 0.406512 & 0.355452 & 0.367612 \\
$\mathbf{0 . 7}$ & 1.303937 & 0.791019 & 0.592413 & $\mathbf{1 . 7}$ & 0.377173 & 0.344349 & 0.361751 \\
$\mathbf{0 . 8}$ & 1.082003 & 0.660111 & 0.518003 & $\mathbf{1 . 8}$ & 0.352354 & 0.334774 & 0.356501 \\
$\mathbf{0 . 9}$ & 0.912858 & 0.570714 & 0.471086 & $\mathbf{1 . 9}$ & 0.331126 & 0.326368 & 0.351732 \\
$\mathbf{1}$ & 0.782375 & 0.508089 & 0.440167 & $\mathbf{2}$ & 0.312777 & 0.318873 & 0.347354 \\
\hline
\end{tabular}

The population of infected mosquitoes decreases as the human recovery rate, $\alpha$, increases.

Table 3.4 The numbers of infected human $x(t)$ at time $t$ for different values of $\alpha$ when $\beta=1_{s} \sigma=0.5$ and $v=1$

\begin{tabular}{cccccccc}
\hline $\boldsymbol{t}$ & $\boldsymbol{\alpha}=\mathbf{2}$ & $\boldsymbol{\alpha}=\mathbf{4}$ & $\boldsymbol{\alpha}=\mathbf{6}$ & $\boldsymbol{t}$ & $\boldsymbol{\alpha}=\mathbf{2}$ & $\boldsymbol{\alpha}=\mathbf{4}$ & $\boldsymbol{\alpha}=\mathbf{6}$ \\
\hline $\mathbf{0}$ & 5 & 5 & 5 & & & & \\
$\mathbf{0 . 1}$ & 3.096693 & 4.141766 & 5.159287 & $\mathbf{1 . 1}$ & 1.22183 & 2.132455 & 2.943887 \\
$\mathbf{0 . 2}$ & 2.433385 & 3.770204 & 5.030365 & $\mathbf{1 . 2}$ & 1.154859 & 2.011282 & 2.786302 \\
$\mathbf{0 . 3}$ & 2.106858 & 3.515945 & 4.809043 & $\mathbf{1 . 3}$ & 1.092208 & 1.9009 & 2.645322 \\
$\mathbf{0 . 4}$ & 1.905678 & 3.299549 & 4.55078 & $\mathbf{1 . 4}$ & 1.033643 & 1.800617 & 2.519376 \\
$\mathbf{0 . 5}$ & 1.760408 & 3.099739 & 4.281572 & $\mathbf{1 . 5}$ & 0.978959 & 1.709667 & 2.406918 \\
$\mathbf{0 . 6}$ & 1.643581 & 2.911027 & 4.016443 & $\mathbf{1 . 6}$ & 0.927963 & 1.627265 & 2.306491 \\
$\mathbf{0 . 7}$ & 1.543002 & 2.732678 & 3.764196 & $\mathbf{1 . 7}$ & 0.880461 & 1.552639 & 2.216749 \\
$\mathbf{0 . 8}$ & 1.45284 & 2.565212 & 3.529557 & $\mathbf{1 . 8}$ & 0.836257 & 1.485047 & 2.136476 \\
$\mathbf{0 . 9}$ & 1.370161 & 2.409216 & 3.314567 & $\mathbf{1 . 9}$ & 0.795153 & 1.423795 & 2.064581 \\
$\mathbf{1}$ & 1.293436 & 2.264983 & 3.119567 & $\mathbf{2}$ & 0.756952 & 1.368241 & 2.000098 \\
\hline
\end{tabular}

The population of infected human increases as the human recovery rate, $\alpha$, increases. 
Table 3.5 The numbers of infected mosquitoes $y(t)$ at time $t$ for different values of $\sigma$ when $\beta=1, \alpha=0.5$ and $v=1$

\begin{tabular}{cccccccc}
\hline $\boldsymbol{t}$ & $\boldsymbol{\sigma}=\mathbf{2}$ & $\boldsymbol{\sigma}=\mathbf{1}$ & $\boldsymbol{\sigma}=\mathbf{0 . 5}$ & $\boldsymbol{t}$ & $\boldsymbol{\sigma}=\mathbf{2}$ & $\boldsymbol{\sigma}=\mathbf{1}$ & $\boldsymbol{\sigma}=\mathbf{0 . 5}$ \\
\hline $\mathbf{0}$ & 5 & 5 & 5 & & & & \\
$\mathbf{0 . 1}$ & 3.096693 & 3.086413 & 3.066167 & $\mathbf{1 . 1}$ & 1.22183 & 1.279857 & 1.379087 \\
$\mathbf{0 . 2}$ & 2.433385 & 2.418203 & 2.389287 & $\mathbf{1 . 2}$ & 1.154859 & 1.224088 & 1.340409 \\
$\mathbf{0 . 3}$ & 2.106858 & 2.093106 & 2.068181 & $\mathbf{1 . 3}$ & 1.092208 & 1.172619 & 1.305692 \\
$\mathbf{0 . 4}$ & 1.905678 & 1.896816 & 1.882173 & $\mathbf{1 . 4}$ & 1.033643 & 1.125116 & 1.274505 \\
$\mathbf{0 . 5}$ & 1.760408 & 1.758466 & 1.757298 & $\mathbf{1 . 5}$ & 0.978959 & 1.081292 & 1.246489 \\
$\mathbf{0 . 6}$ & 1.643581 & 1.649905 & 1.663979 & $\mathbf{1 . 6}$ & 0.927963 & 1.040888 & 1.221329 \\
$\mathbf{0 . 7}$ & 1.543002 & 1.558567 & 1.588933 & $\mathbf{1 . 7}$ & 0.880461 & 1.003657 & 1.198745 \\
$\mathbf{0 . 8}$ & 1.45284 & 1.478374 & 1.52566 & $\mathbf{1 . 8}$ & 0.836257 & 0.969364 & 1.178484 \\
$\mathbf{0 . 9}$ & 1.370161 & 1.406202 & 1.470748 & $\mathbf{1 . 9}$ & 0.795153 & 0.937786 & 1.160314 \\
$\mathbf{1}$ & 1.293436 & 1.340357 & 1.422277 & $\mathbf{2}$ & 0.756952 & 0.908708 & 1.144024 \\
\hline
\end{tabular}

The population of infected mosquitoes increases as the number of female mosquitoes per human host in an infection free steady state $\sigma$ increases.

Table 3.6 The numbers of infected human $x$ (t) at time $t$ for different values of $\sigma$ when $\beta=1, \alpha=2$ and $v=1$

\begin{tabular}{cccccccc}
\hline $\boldsymbol{t}$ & $\boldsymbol{\sigma}=\mathbf{2}$ & $\boldsymbol{\sigma}=\mathbf{1}$ & $\boldsymbol{\sigma}=\mathbf{0 . 5}$ & $\boldsymbol{t}$ & $\boldsymbol{\sigma}=\mathbf{2}$ & $\boldsymbol{\sigma}=\mathbf{1}$ & $\boldsymbol{\sigma}=\mathbf{0 . 5}$ \\
\hline $\mathbf{0}$ & 10 & 10 & 10 & & & & \\
$\mathbf{0 . 1}$ & 6.307438 & 6.462039 & 6.770258 & $\mathbf{1 . 1}$ & 0.680533 & 0.971118 & 1.563502 \\
$\mathbf{0 . 2}$ & 4.461663 & 4.686564 & 5.133346 & $\mathbf{1 . 2}$ & 0.600122 & 0.887358 & 1.477027 \\
$\mathbf{0 . 3}$ & 3.31832 & 3.580747 & 4.101029 & $\mathbf{1 . 3}$ & 0.535898 & 0.819774 & 1.406901 \\
$\mathbf{0 . 4}$ & 2.544652 & 2.827539 & 3.388144 & $\mathbf{1 . 4}$ & 0.484005 & 0.764596 & 1.349483 \\
$\mathbf{0 . 5}$ & 1.997105 & 2.290648 & 2.872949 & $\mathbf{1 . 5}$ & 0.441587 & 0.719023 & 1.302033 \\
$\mathbf{0 . 6}$ & 1.598968 & 1.897315 & 2.490474 & $\mathbf{1 . 6}$ & 0.406512 & 0.680951 & 1.262477 \\
$\mathbf{0 . 7}$ & 1.303937 & 1.603549 & 2.201215 & $\mathbf{1 . 7}$ & 0.377173 & 0.648793 & 1.229229 \\
$\mathbf{0 . 8}$ & 1.082003 & 1.38075 & 1.979249 & $\mathbf{1 . 8}$ & 0.352354 & 0.62134 & 1.201067 \\
$\mathbf{0 . 9}$ & 0.912858 & 1.209481 & 1.806775 & $\mathbf{1 . 9}$ & 0.331126 & 0.597667 & 1.177043 \\
$\mathbf{1}$ & 0.782375 & 1.076162 & 1.671212 & $\mathbf{2}$ & 0.312777 & 0.577058 & 1.156417 \\
\hline
\end{tabular}

The population of infected human increases as the number of female mosquitoes per human host in an infection free steady state $\sigma$ increases.

Table 3.7 The numbers of infected mosquitoes $y(t)$ at time $t$ for different values of $v$ when $\alpha=2, \sigma=0.5$ and $\beta=1$

\begin{tabular}{cccccccc}
\hline $\boldsymbol{t}$ & $\boldsymbol{v}=\mathbf{5}$ & $\boldsymbol{v}=\mathbf{3}$ & $\boldsymbol{v}=\mathbf{1}$ & $\boldsymbol{t}$ & $\boldsymbol{v}=\mathbf{5}$ & $\boldsymbol{v}=\mathbf{3}$ & $\boldsymbol{v}=\mathbf{1}$ \\
\hline $\mathbf{0}$ & 5 & 5 & 5 & & & & \\
$\mathbf{0 . 1}$ & 3.096693 & 3.479679 & 3.746678 & $\mathbf{1 . 1}$ & 1.22183 & 1.208336 & 1.350807 \\
$\mathbf{0 . 2}$ & 2.433385 & 2.989026 & 3.335254 & $\mathbf{1 . 2}$ & 1.154859 & 1.100999 & 1.226213 \\
$\mathbf{0 . 3}$ & 2.106858 & 2.665655 & 3.005673 & $\mathbf{1 . 3}$ & 1.092208 & 1.004466 & 1.113951 \\
$\mathbf{0 . 4}$ & 1.905678 & 2.399594 & 2.713186 & $\mathbf{1 . 4}$ & 1.033643 & 0.917558 & 1.012737 \\
$\mathbf{0 . 5}$ & 1.760408 & 2.166862 & 2.450933 & $\mathbf{1 . 5}$ & 0.978959 & 0.839229 & 0.921423 \\
$\mathbf{0 . 6}$ & 1.643581 & 1.959877 & 2.21546 & $\mathbf{1 . 6}$ & 0.927963 & 0.768551 & 0.838982 \\
$\mathbf{0 . 7}$ & 1.543002 & 1.774897 & 2.003929 & $\mathbf{1 . 7}$ & 0.880461 & 0.704699 & 0.764497 \\
$\mathbf{0 . 8}$ & 1.45284 & 1.609295 & 1.813829 & $\mathbf{1 . 8}$ & 0.836257 & 0.646943 & 0.69715 \\
$\mathbf{0 . 9}$ & 1.370161 & 1.460897 & 1.642913 & $\mathbf{1 . 9}$ & 0.795153 & 0.594635 & 0.636208 \\
$\mathbf{1}$ & 1.293436 & 1.327804 & 1.489172 & $\mathbf{2}$ & 0.756952 & 0.547201 & 0.581018 \\
\hline
\end{tabular}

The population of infected mosquitoes decreases as per capita death rate of mosquitoes, $v$, increases. 
Table 3.8 The numbers of infected human $x(t)$ at time $t$ for different values of $v$ when $\alpha=2, \sigma=0.5$ and $\beta=1$

\begin{tabular}{cccccccc}
\hline $\boldsymbol{t}$ & $\boldsymbol{v}=\mathbf{5}$ & $\boldsymbol{v}=\mathbf{3}$ & $\boldsymbol{v}=\mathbf{1}$ & $\boldsymbol{t}$ & $\boldsymbol{v}=\mathbf{5}$ & $\boldsymbol{v}=\mathbf{3}$ & $\boldsymbol{v}=\mathbf{1}$ \\
\hline $\mathbf{0}$ & 10 & 10 & 10 & & & & \\
$\mathbf{0 . 1}$ & 6.307438 & 2.310349 & 0.581018 & $\mathbf{1 . 1}$ & 0.680533 & 0.096826 & 0.059375 \\
$\mathbf{0 . 2}$ & 4.461663 & 0.744322 & 0.155376 & $\mathbf{1 . 2}$ & 0.600122 & 0.093137 & 0.057117 \\
$\mathbf{0 . 3}$ & 3.31832 & 0.320288 & 0.085613 & $\mathbf{1 . 3}$ & 0.535898 & 0.08951 & 0.054846 \\
$\mathbf{0 . 4}$ & 2.544652 & 0.18891 & 0.075494 & $\mathbf{1 . 4}$ & 0.484005 & 0.085931 & 0.052573 \\
$\mathbf{0 . 5}$ & 1.997105 & 0.142982 & 0.072395 & $\mathbf{1 . 5}$ & 0.441587 & 0.0824 & 0.050307 \\
$\mathbf{0 . 6}$ & 1.598968 & 0.124335 & 0.070207 & $\mathbf{1 . 6}$ & 0.406512 & 0.078924 & 0.048057 \\
$\mathbf{0 . 7}$ & 1.303937 & 0.115053 & 0.068112 & $\mathbf{1 . 7}$ & 0.377173 & 0.075509 & 0.045832 \\
$\mathbf{0 . 8}$ & 1.082003 & 0.10919 & 0.065987 & $\mathbf{1 . 8}$ & 0.352354 & 0.072164 & 0.043639 \\
$\mathbf{0 . 9}$ & 0.912858 & 0.104641 & 0.063819 & $\mathbf{1 . 9}$ & 0.331126 & 0.068896 & 0.041487 \\
$\mathbf{1}$ & 0.782375 & 0.100621 & 0.061612 & $\mathbf{2}$ & 0.312777 & 0.065711 & 0.039381 \\
\hline
\end{tabular}

The population of infected human increases and later decreases as per capita death rate of mosquitoes $v$ increases.

\section{Conclusion}

A simple malaria model that captures the essential elements of malaria epidemiology has been analyzed and investigated, resulting in a system of ordinary differential equations (ODEs). The simulations were conducted using the Runge-Kutta integration scheme with a modified version of Newton-Raphson shooting method with $\beta$ (the per capita biting rate of mosquitoes on human), $\alpha$ (the human recovery rate), $\sigma$ (the number of female mosquitoes per human host in an infection free steady state) and $v$ (the per capita death rate of mosquitoes) as prescribed parameters. The results obtained showed that the parameters involved play a crucial role in the interaction between infected human and infected mosquito. The mathematical modeling of malaria epidemiology as carried out in this research work would go a long way in assisting governmental bodies and health administrators in establishing curative and preventive control measures towards the eradication of malaria.

\section{References}

[1] R. Ross, The prevention of malaria (London: John Murray, 1911).

[2] G. Macdonald, The epidemiology and control of malaria (London: Oxford University Press, 1957).

[3] J. L. Aron and R. M. May, The population dynamics of malaria, in R. M. Anderson (Ed.), The population dynamics of infectious disease: theory and applications, (London: Chapman and Hall, 1982), 139-179.

[4] R. M. Anderson and R. M. May, Infectious diseases of humans: dynamics and control (Oxford: Oxford Unversity Press, 1991).

[5] M. G. Roberts and J. A. P. Heesterbeek, A new method for estimating the effort required to control an infectious disease, Proc. 270th Royal Society of London Series B, London, 2003, 1359-1364.

[6] N. R. Chitnis, Using mathematical models in controlling the spread of malaria, doctoral diss., the University of Arizona, Tucson, AZ, 2005. 